\title{
Developing a Crew Time Model for Human Exploration Missions to Mars
}

\author{
Bryan Mattfeld \& Chel Stromgren \\ Binera, Inc. \\ 8455 Colesville Road, Suite 1075 \\ Silver Spring, MD,20910 \\ 301-579-5242 \\ b.mattfeld@binera.com, \\ c.stromgren@binera.com
}

\author{
Hilary Shyface \\ Analytical Mechanics Associates, \\ Inc. \\ 21 Enterprise Parkway, Suite 300 \\ Hampton, VA 23666 \\ 757-864-2120 \\ h.shyface@ama-inc.com
}

\author{
William Cirillo \& Kandyce Goodliff \\ NASA Langley Research Center \\ Hampton, VA 23681 \\ 757-864-1938, 757-864-4969 \\ william.m.cirillo@nasa.gov, \\ kandyce.e.goodliff@nasa.gov
}

Candidate human missions to Mars require mission lengths that could extend beyond those that have previously been demonstrated during crewed Lunar (Apollo) and International Space Station (ISS) missions. The nature of the architectures required for deep space human exploration will likely necessitate major changes in how crews operate and maintain the spacecraft. The uncertainties associated with these shifts in mission constructs - including changes to habitation systems, transit durations, and system operations - raise concerns as to the ability of the crew to complete required overhead activities while still having time to conduct a set of robust exploration activities.

This paper will present an initial assessment of crew operational requirements for human missions to the Mars surface. The presented results integrate assessments of crew habitation, system maintenance, and utilization to present a comprehensive analysis of potential crew time usage. Destination operations were assessed for a short ( 50 day) and long duration ( $\sim 500$ day) surface habitation case. Crew time allocations are broken out by mission segment, and the availability of utilization opportunities was evaluated throughout the entire mission progression.

To support this assessment, the integrated crew operations model (ICOM) was developed. ICOM was used to parse overhead, maintenance and system repair, and destination operations requirements within each mission segment outbound transit, Mars surface duration, and return transit to develop a comprehensive estimation of exploration crew time allocations. Overhead operational requirements included daily crew operations, health maintenance activities, and down time. Maintenance and repair operational allocations are derived using the Exploration Maintainability and Analysis Tool (EMAT) to develop a probabilistic estimation of crew repair time necessary to maintain systems functionality throughout the mission.

\section{TABLE OF CONTENTS}

1. INTRODUCTION .1

2. MODEL OVERVIEW............................................2

3. DESCRIPTION OF CASES .....................................5

4. ASSESSMENT RESULTS .................................66

6. CONCLUSIONS ..........................................12

7. FUTURE WORK ........................................13

ACKNOWLEDGEMENTS....................................14

REFERENCES............................................................14

BIOGRAPHY .....................................................15

\section{INTRODUCTION}

Historically, on the International Space Station, there has been a challenge in allocating a significant amount of time for the completion of science and other types of utilization. The time required for other activities, including crew downtime, maintenance, public relations, and other work items consumes a large fraction of the total available crew time.

Crewed missions to Mars will involve crew operations that are significantly different from all past crewed space missions. Not only will the durations be substantially longer, but also the missions will involve an increased level of autonomy in operations and maintenance, and increased healthcare/exercise requirements. All of these changes have the potential to increase time demands on the crew.

Because Mars missions are potentially more challenging than ISS from a crew time perspective, there is concern that the crew may not be able to allocate sufficient time to utilization activities, given the other demands on their time. In addition, there is a concern that critical activities, such as maintenance and repair might impose time demands on the crew that are inconsistent with physiological and psychological wellbeing.

This paper describes an effort to evaluate potential crew time demands for different Mars missions. As part of this effort, the authors developed a statistical crew time allocation tool that determines required time demands for the crew and then determines the amount of marginal time available for utilization activities, if any. The model also identifies cases where crew flight rules regarding work day limitations may be exceeded. The model specifically evaluates in-space as well as surface mission segments for all missions and segregates IVA and EVA activities.

The analysis is driven by historical data collected from ISS crew increments. This data was used to identify time allocations for each required crew activities and to assess the variance between periods and between different crew members. The rich historical data provides a strong basis for estimating time requirements for certain required activities.

Other crew time drivers, such as maintenance and repair tasks were developed using a detailed simulation model of 
the exploration elements. Because Mars exploration hardware is liable to be significantly different from ISS systems, the expected maintenance and repair activities may be different from those experienced on ISS. The simulation provides probability distributions for required repair time, specific to the exploration systems, which are integrated into the crew time model.

This paper will present a detailed description of the Integrated Crew Time Model. It will then present the Mars missions cases that were analyzed. The baseline results of the crew time assessment will then be presented, including availably of time for utilization activities during different missions and mission segments. Finally, a comprehensive sensitivity analysis will be presented that indicates how improvement in certain crew time requirements impact the amount of utilization time that is available.

\section{Model OVERVIEW}

The Integrated Crew Operations Model (ICOM) is an integrated scenario-based analysis model that incorporates time requirements for daily crew operations, repair, and destination exploration to produce detailed crew operational allocation estimates for space exploration missions. The scenario-based modeling environment utilizes an architecture driven framework to develop crew time results specifically tailored to various crewed exploration missions. Analysis is conducted over each mission segment outbound transit, duration at destination, and return transit to capture variations in operational requirements. ICOM can be run bi-directionally, either to forward solve for utilization time availability given mission constraints or in reverse to define the crew operations performance thresholds to achieve destination utilization targets.

The model combines the full set of baseline mission inputs, crew time liens, maintenance requirements, excursion architecture assumptions, EVA schedules, and setup/offloading needs to produce a single integrated crew operations estimate.

\section{Mission Segments}

Mission segments may be designated within the model to allow for independent definition of governing assumptions, crew capabilities, and operational objectives. Crew time solutions are derived for each individual mission segment. Systems definition, fixed cargo elements, and exploration capabilities may be varied per phase to capture architectural variations. Each mission segment is given a defined duration, which is based on the mission profile.

\section{Crew Time Liens}

Crew time liens represent all crew time for activities not directly related to utilization or maintenance. This includes activities required to keep the crew healthy, training and conference, and general upkeep of the spacecraft. Lien estimates are derived from historical ISS crew time data from the years 2007 through 2013. [1] Each ISS day over this period was analyzed on a per-crew, per-day basis to develop a statistical baseline from which the model could be adjusted to account for specific mission characteristics. Table 1 provides a detailed list of the crew time lien categories and the associated tasks.

Table 1: Crew Liens

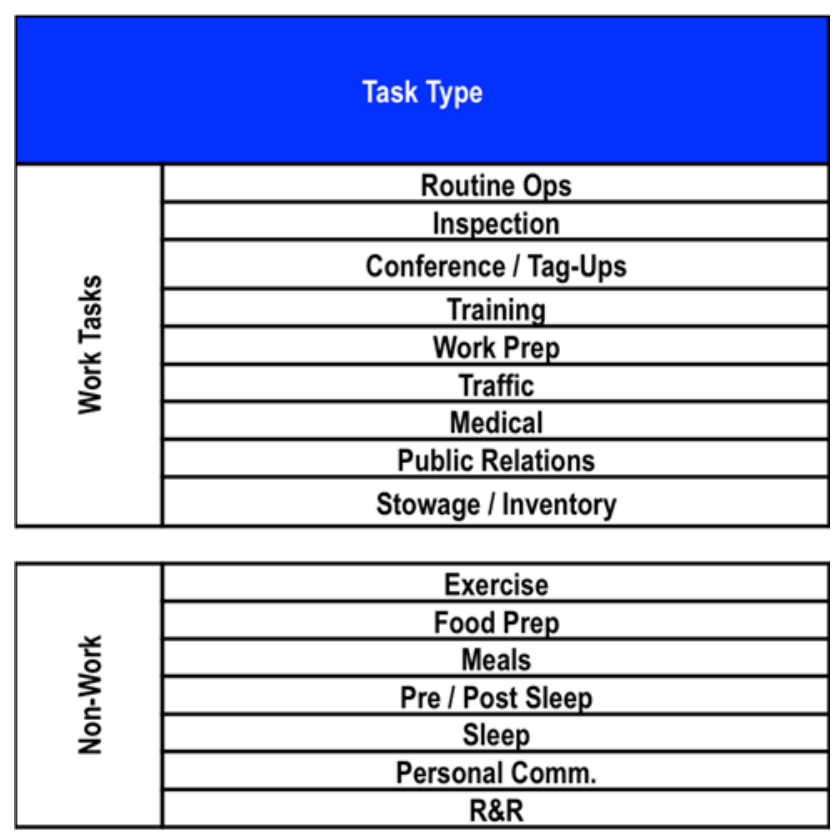

When forward solving for mission utilization availability, time liens are rigid constraints within the model. If a utilization target is specified, the model will instead return the required improvements in crew operations that are necessary to achieve the prescribed utilization threshold. Lien tasks may be prioritized for these cases, so that the model will attempt to maintain nominal operations for the highest priority tasks.

\section{Repair and Maintenance}

Initial repair and maintenance time estimates are supplied to ICOM via the Exploration Maintainability Analysis Tool (EMAT) [2]. EMAT utilizes a comprehensive model of crew critical exploration systems and reliability estimates that assume an evolutionary development from current ISS systems. System models were built up from the component level given direct input of subject matter experts. Table 2 provides the primary exploration systems included in the maintainability assessment. 
Table 2: Exploration Systems List

\begin{tabular}{|c|c|}
\hline Exploration System & Category \\
\hline Carbon Dioxide Removal & ECLSS \\
\hline Carbon Dioxide Reduction & ECLSS \\
\hline $\begin{array}{c}\text { Oxygen \& Nitrogen Supply and } \\
\text { Distribution }\end{array}$ & ECLSS \\
\hline Oxygen Generation System & ECLSS \\
\hline Air Temperature and Humidity \\
Control & ECLSS \\
\hline Air Circulation \& Ventilation & ECLSS \\
\hline Trace Contaminant Removal & ECLSS \\
\hline Pressure Control and Relief & ECLSS \\
\hline Atmospheric Constituents Monitoring & ECLSS \\
\hline Water Recovery \& Processing & ECLSS \\
\hline Communications \& Tracking & Navigation and Communications \\
\hline Attitude \& Rate Determination & Navigation and Communications \\
\hline Electrical Power System & EPS \\
\hline Command \& Data Handling & C\&DH \\
\hline Active Thermal Control & TCS \\
\hline Fire Detection and Suppression & Periodic Maintenance \\
\hline Food System & Periodic Maintenance \\
\hline Exercise & Periodic Maintenance \\
\hline Medical & Periodic Maintenance \\
\hline Solid Waste Removal & Periodic Maintenance \\
\hline Laundry & Periodic Maintenance \\
\hline
\end{tabular}

Systems maintenance and repair time requirements are probabilistically generated in a Monte Carlo environment by simulating system failure events and maintenance activities on a day-by-day basis during each mission segment. ISS heritage crew time to repair (CTTR) data is used to generate the expected crew time required to repair each failure event. It is assumed all systems are fully operational at the beginning of the mission and for each failure event the mission is outfitted with all necessary spare parts and repair tools. Repair and maintenance times are taken from the EMAT results at the $90 \%$ confidence level.

The results generated by EMAT represent the expected crew time allocation required to maintain sufficient system functionality to support crew life for the entirety of each mission phase. The maintenance times developed in EMAT represent repair operations as conducted on ISS. It may however, be necessary to account for additional factors for an extended exploration mission, such as progressive crew fatigue or performance degradation. These types of corrections are made once the initial maintenance and repair time estimates are passed to ICOM, where statistical adjustments may be made to better capture mission features. The resultant modified maintenance and repair time datasets are then used within the model to determine crew operations. The interactions between EMAT and ICOM are given in Figure 1 below.

All component reliability data - mean time between failures (MTBFs), K-factors, and CTTRs - are derived from historical ISS performance data. Detailed descriptions and operational dependencies (along with example schematic diagrams) of each exploration system model included in this study, along with a functional description of EMAT, are described in further detail in the 2013 Assessment of Maintainability for Future Human Asteroid and Mars Missions. [3]

\section{Destination Operations and Surface Traversal}

Mobility capabilities are critical to enabling utilization opportunities for Mars destination missions and must be properly characterized to correctly model crewed destination operations. ICOM utilizes a three-phase excursion methodology to model two primary crewed surface activities: local exploration/set-up tasks and longdistance traversal and exploration. Both surface activity types are defined by specifying excursion duration, preparation and analysis periods, and post-excursion rest requirements. Figure 2 details how surface activities characterization is structured within the model.

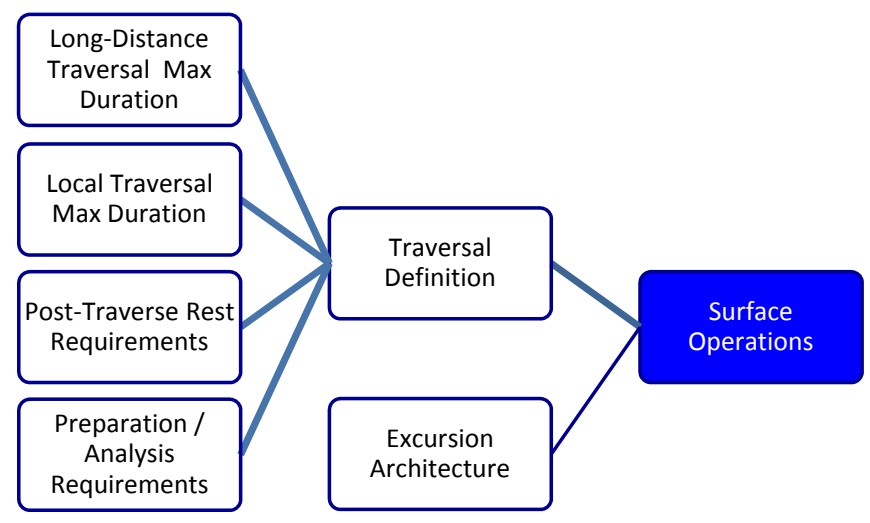

Figure 2: Surface Operations Definition

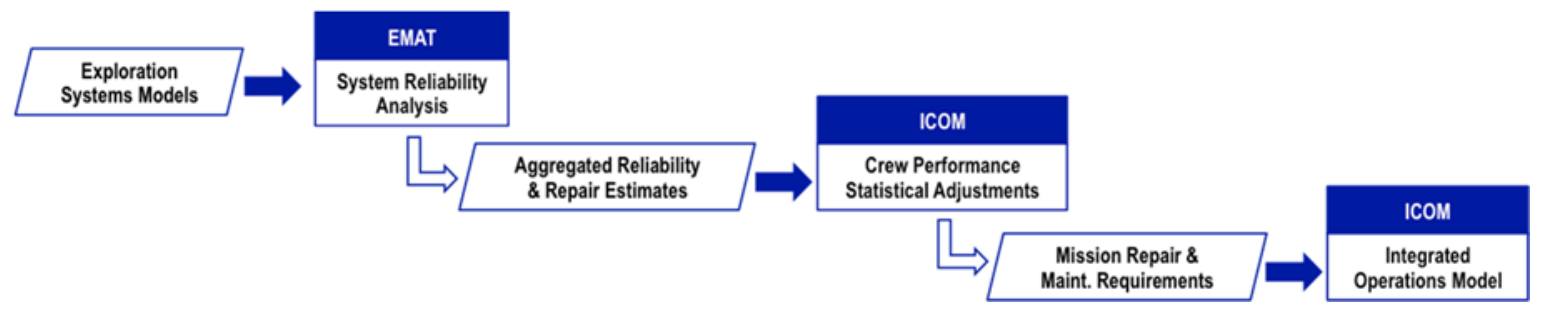

Figure 1: Model Data Flow 
Within each excursion phase, additional prioritizations and constraints are set to define target crew operations during long-distance traversal. Pressurized rovers are used to conduct the long-distance traversals. Note that baseline EVA assumptions may be overridden for EVA during excursions and excursion EVA are independent of outpost EVA solutions. In the baseline model, the following allocations may be prioritized and threshold constraints may be defined:

- $\quad$ Pressurized Rover Driving Time

- $\quad$ Excursion EVA Rate

- Excursion IVA Utilization Targets

- $\quad$ EVA Maintenance Requirements

- $\quad$ Local Setup Requirements

Using this prioritization structure, along with the segment definitions described prior, a routine is executed to determine total excursion period availability. Given mission duration parameters, ICOM will solve for the total number of excursion activity cycles that could be achieved during the crewed surface duration. Once the total number of available excursion cycles is defined, the model will optimize excursion activities in an attempt to achieve exploration goals, assuming critical lien requirements are satisfied. Any additional crewed excursion time is allocated to the completion of remaining liens during the excursion. If the excursion crew is not able to complete the total allocation of remaining liens, certain non-critical tasks may be made up at the surface outpost after the excursion is complete. Non-critical tasks include training, medical, personal communications, stowage, and inventory management.

The excursion methodology used by ICOM assumes that there are no repair and maintenance activities performed during excursions beyond critical fixes to enable crew return to the local habitat in the event of a failure. While excursion cycles are assumed to require continuous blocks of crew time, individual tasks within each excursion event do not require continuity. For mission architectures in which only a portion of the surface crew occupies the pressurized rovers at a given time, it is expected that the crew remaining within the outpost have the capability to maintain full outpost operations.

\section{EVA}

Within ICOM, crewed EVA is split into two activity types, work and utilization tasks. Work EVA hours are treated as high priority time liens that the crew must complete as part of standard operations. Baseline contributors to work EVA include external maintenance and repair, surface systems setup, and destination offloading or logistics transfer. Utilization EVA is generally treated as a dependent variable that is to be maximized during excursions for long-stay missions and at the habitat for short-stay missions. When evaluating EVA operations during surface mission segments, outpost and excursion activities are handled as independent activity classes, since excursion EVA are focused on utilization. EVAs during transit operations are allocated only for maintenance and repair. EVA time requirements are evaluated exclusively within each mission segment. EVA performance assumptions are defined based on maximum frequency, suit maintenance requirements, and post-activity crew rest requirements.

EVA suit maintenance requirements are specifically evaluated as part of the crew time model. Suit maintenance is dynamically modeled within ICOM based on both usage rate and overall EVA systems lifetime. Because suit maintenance requirements may be usage-dependent, an iterative routine is employed to maximize total available EVA hours while still fitting within the total available crew time. Crew EVA hours are incrementally built-up by solving for suit maintenance requirements for a given EVA time allocation and then summing the total EVA and suit maintenance hours. If this interim result remains within the allowable total crew time, the process is repeated by adding additional EVA hours and once again solving for maintenance requirements. This process is continued until crew EVA hours are maximized but still fit within total crew time constraints.

Additional assumptions, such as maximum EVA duration and weekly activity limits are also considered during this iterative procedure. The solution process will ensure that the combined activity allocation of both work and utilization tasks will never exceed crew and EVA systems capability thresholds set for the mission segment.

\section{Setup and Offloading}

Set-up and offloading requirements are modeled as single event operational time that must be accomplished with high priority in the mission segment to which they are assigned. Fixed cargo elements are entered into the model with associated outfitting/setup IVA and unloading/setup EVA time requirements. Time estimates are expected to cover all off-loading, transport, installation, and outfitting operations associated with each fixed element. Continuous time allocation is not required to complete setup and offloading tasks; it is assumed the crew may efficiently allocate time given daily availability. 


\section{DESCRIPTION OF CASES}

\section{Mission Trajectory and Segment Durations}

Mission durations were developed in accordance with NASA Human Spaceflight Architecture Team's (HAT) Mars surface destination team assumptions. A conjunction class trajectory with the total crew duration of 1100 days was assumed for all cases. All cases assumed a crew of four with all crew members descending to the surface. Long stay surface durations were limited to 460 days. Short-stay architectures assumed surface durations of up to 50 days. Mars destination and Earth return transit times were assumed relatively symmetric to simplify model assumptions, with 320 days allocated to each transit period. Both cases involved transit segments totaling 640 days with 460 days in the vicinity of Mars. For the short-stay architectures, the additional time that the crew spends in Mars vicinity while not on the surface is treated identically to the in-transit portions of the mission.

\section{Crew Operational Liens}

Operational liens were derived from historical ISS data, adjusted to reflect transit and surface mission architectures. Table 3 provides a detailed list of crew lien assumptions, including the ISS baseline values. Values were modified (shown with green background) to reflect changes in system architecture, increased crew autonomy, and extended crew exposure to the deep space environment. Lien times do not include crew time allocated for system maintenance/repair or utilization activities.

Numerous work tasks were adjusted to better represent the increased autonomy required for crew operations. Training and work preparation activities were both increased substantially to reflect the types of complex exploration systems that the crew will be responsible for autonomously maintaining. Traffic time allocations were removed, given the absence of crew handover and cargo resupply docking/undocking activities during the exploration mission. The application of RFID technologies and the lack of re-supply should enable increased efficiency of cargo handling and thus reduce the crew time required.

Exercise requirements during Mars transit were assumed to increase over current ISS standards. With total transit durations that may exceed 600 days, the crew will be exposed to a microgravity envrionment for durations nearly $400 \%$ longer than current ISS experience. At Mars surface, a reduced exercise requirement was assumed. The combination of the hypo gravity environment and an increased EVA rate will somewhat reduce the need for additional exercise. Simplified, MRE style meals will reduce crew time required to prepare food.

Table 3: Crew Lien Time Assumptions

\begin{tabular}{|c|c|c|c|c|}
\hline & Task Type & $\begin{array}{c}\text { Average Weekly } \\
\text { ISS Time (Hours/ } \\
\text { Week/Crew) }\end{array}$ & $\begin{array}{c}\text { Mars Transit Time } \\
\text { (Hours/Week/ } \\
\text { Crew) }\end{array}$ & $\begin{array}{l}\text { Mars Surface } \\
\text { Time (Hours/ } \\
\text { Week/Crew) }\end{array}$ \\
\hline \multirow{9}{*}{ 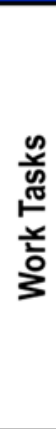 } & Routine Ops & 4.0 & 4.0 & 4.0 \\
\hline & Inspection & 1.0 & 2.0 & 2.0 \\
\hline & Conference / Tag-Ups & 9.0 & 7.0 & 7.0 \\
\hline & Training & 2.5 & 4.0 & 4.0 \\
\hline & Work Prep & 3.5 & 6.0 & 6.0 \\
\hline & Traffic & 2.0 & 0.0 & 0.0 \\
\hline & Medical & 4.0 & 4.0 & 4.0 \\
\hline & Public Relations & 1.5 & 1.5 & 1.5 \\
\hline & Stowage / Inventory & 2.0 & 1.0 & 1.0 \\
\hline \multirow{7}{*}{ 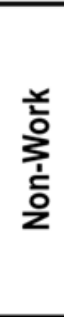 } & Exercise & 16.0 & 18 & 10 \\
\hline & Food Prep & 3.25 & 2.0 & 2.0 \\
\hline & Meals & 12.25 & 12.25 & 12.25 \\
\hline & Pre / Post Sleep & 10.5 & 10.5 & 10.5 \\
\hline & Sleep & 59.5 & 59.5 & 59.5 \\
\hline & Personal Comm. & 1.5 & 1.5 & 1.5 \\
\hline & R\&R & 20.0 & 20.0 & 20.0 \\
\hline
\end{tabular}




\section{Habitat/Outpost Architecture}

The crew of four was assumed to occupy a single, monolithic habitat during outbound and return transit. All consumables necessary for crew survival were assumed carried with the transit habitat; no cargo transfer was necessary during the mission. During surface operations, the transit habitat remained dormant in Mars orbit until the crewed Mars ascent vehicle rendezvous occurred, at which point the crew immediately initiated Earth return.

To assess surface operations, it was assumed all surface capabilities and cargo were pre-deployed and landed prior to crew arrival. The long-stay surface architecture includes a single centrally located, monolithic surface habitat and two Small Pressurized Rovers (SPR). Excursions were assumed to be conducted using the SPRs.

The surface habitat was assumed to contain ECLSS capabilities with a high rate of closure. The primary outpost contains volume and resources to enable science activities. Rover habitation capabilities were limited, providing a maximum two weeks traversal time. SPR capacity is minimal, with only essential fieldwork equipment and life support consumables carried onboard. The short-stay architecture assumed the crew used a pressurized volume within the descent module as the central surface habitat. Surface exploration occurring using Apollo-style unpressurized rovers.

\section{Surface Operations}

Upon crew arrival at Mars surface, it was assumed a hypogravity reconditioning period was required of the crew prior to commencement of surface EVA. The long-duration surface stay required 30 day of reconditioning, with the short duration case requiring 7 day prior to surface operations occurring. During reconditioning periods, minimal physical tasks are performed by the crew.

For the long-duration case, the next 30 days after crew reconditioning were allocated to local exploration and setup operations. During this period, any excursions with the SPR did not exceed 3 day, with a day of rest and a day of analysis occurring after each short excursion. After reconditioning and initial setup, 15-day excursion cycles could occur during the next 400 days at the surface. The last 30 surface days were allocated to final analysis and departure preparations.

Each SPR is driven by a single crewmember during surface excursions, with the additional two crewmembers remaining at the central habitat. It is an operational requirement that two rovers are used for each excursion to provide drive-back capability in event of a failure. Each rover is assumed to be able to drive at an average of $15 \mathrm{~km} / \mathrm{hr}$. Driving time is included as part of the workday.

For the short-duration surface case, 41 days following the initial 7 allotted for reconditioning were eligible for surface operations. During this period, two crewmembers at a time were able to conduct surface EVA and conduct surface exploration using the unpressurized rovers. The final 2 days at the surface are allocated to final analysis and departure preparations.

EVA constraints were developed in accordance with HAT EVA team assumptions. As such, the baseline maximum EVA rate is set at 24 hours per 7 day period, with a full day of rest required to occur in that same 7 day period. For short duration missions, this restriction may be exceeded so that greater than 24 hours of EVA may be performed in 7 days, however after 24 hours of EVA one day of rest must occur.

\section{Assessment Results}

Under the assumptions provided in Section 3, an initial assessment of crew operations was conducted for both short and long surface-stay Mars exploration missions. This assessment included analysis of both transit and surface periods for each mission. The baseline analysis provides insight into crew time restrictions for Mars exploration scenarios and allows for the evaluation of primary operational drivers, identification of potential issues, and to set a statistical benchmark from which sensitivities may be explored. For all cases, utilization time was solved for as the dependent variable in the assessment. Results are presented in Figures 3, 4, 5, 6, and 7. Crew hours are broken out per task type for Mars transit periods and short and long durations on Mars surface.

For both types of missions the crew time available for utilization activities during transit is minimal. For surface durations, there is more utilization time available but the total percentage of time available for such activities is still limited. A substantial portion of total crew time must be allocated to operational liens and maintenance, greatly restricting both IVA and EVA utilization opportunities.

For the nominal 320-day outbound and inbound transit periods, a total of 213 crew hours are available for all utilization activities. This constitutes only $1 \%$ of total transit crew hours for IVA utilization activities. 


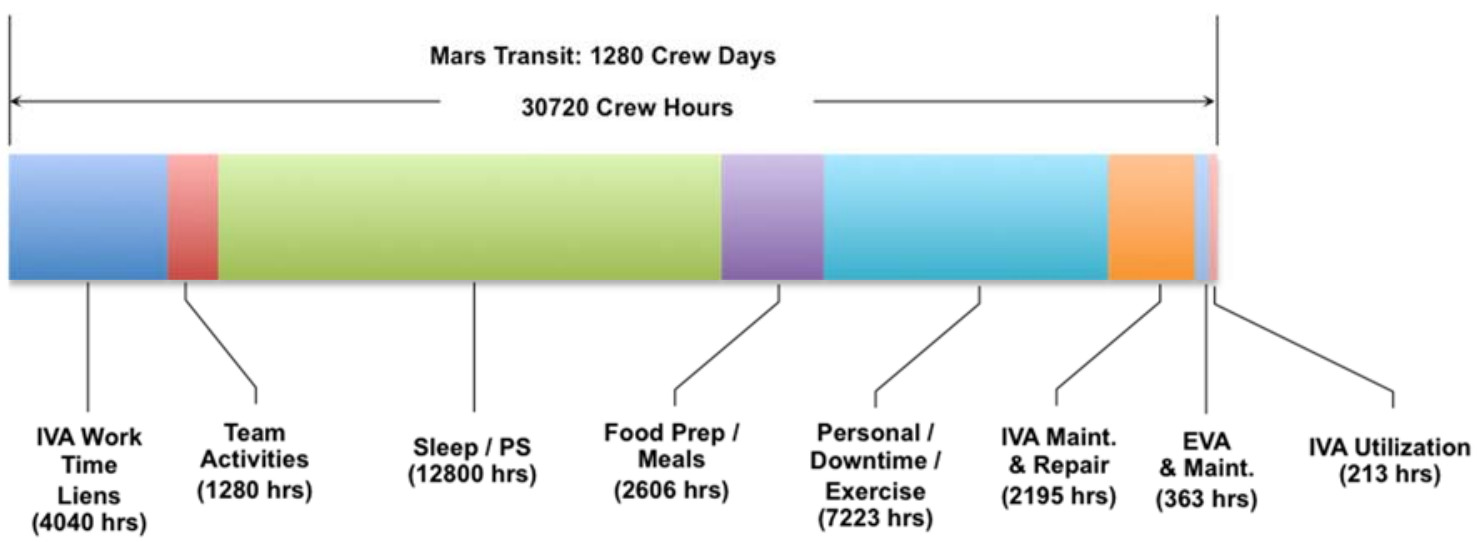

Figure 1: Transit Crew Time Allocations

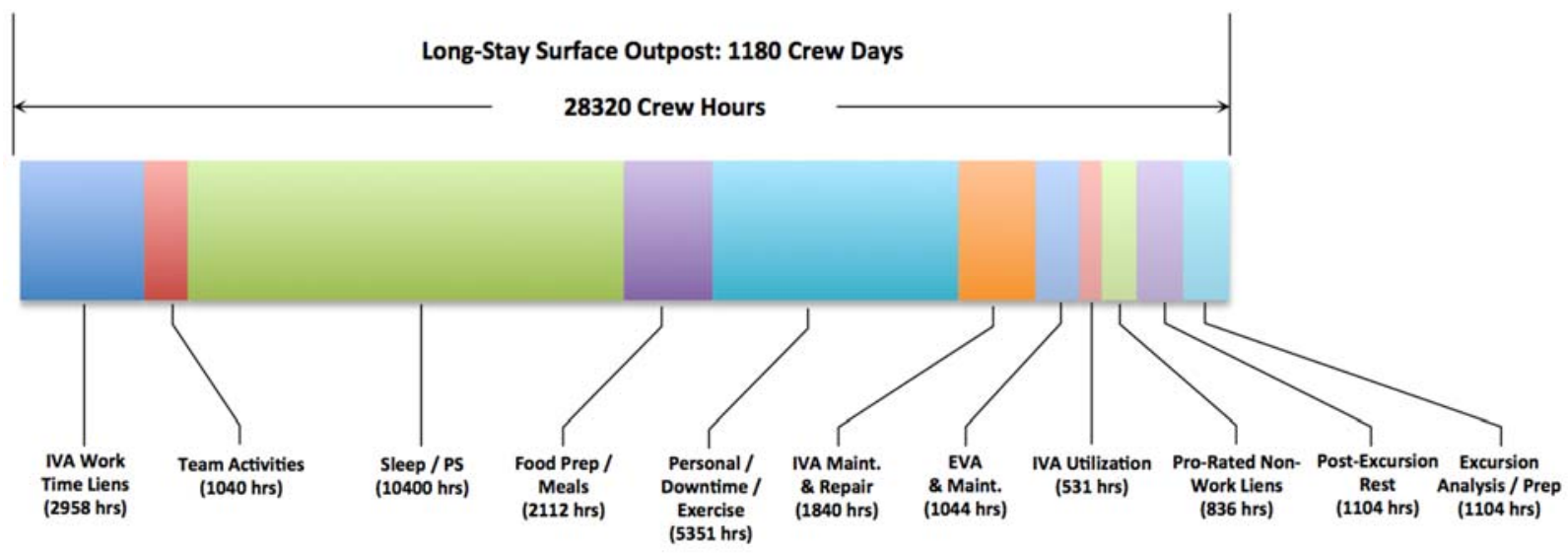

Figure 4: Long-Stay Surface Outpost Crew Time Allocations

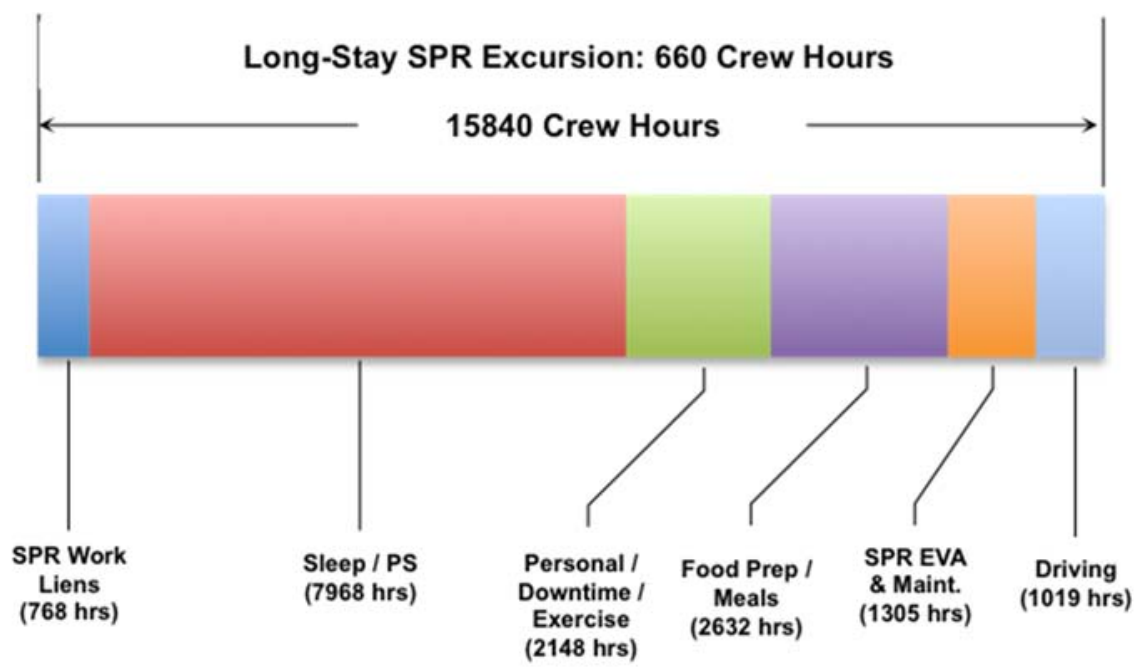

Figure 5: Long-Stay SPR Excursion Crew Time Allocations 


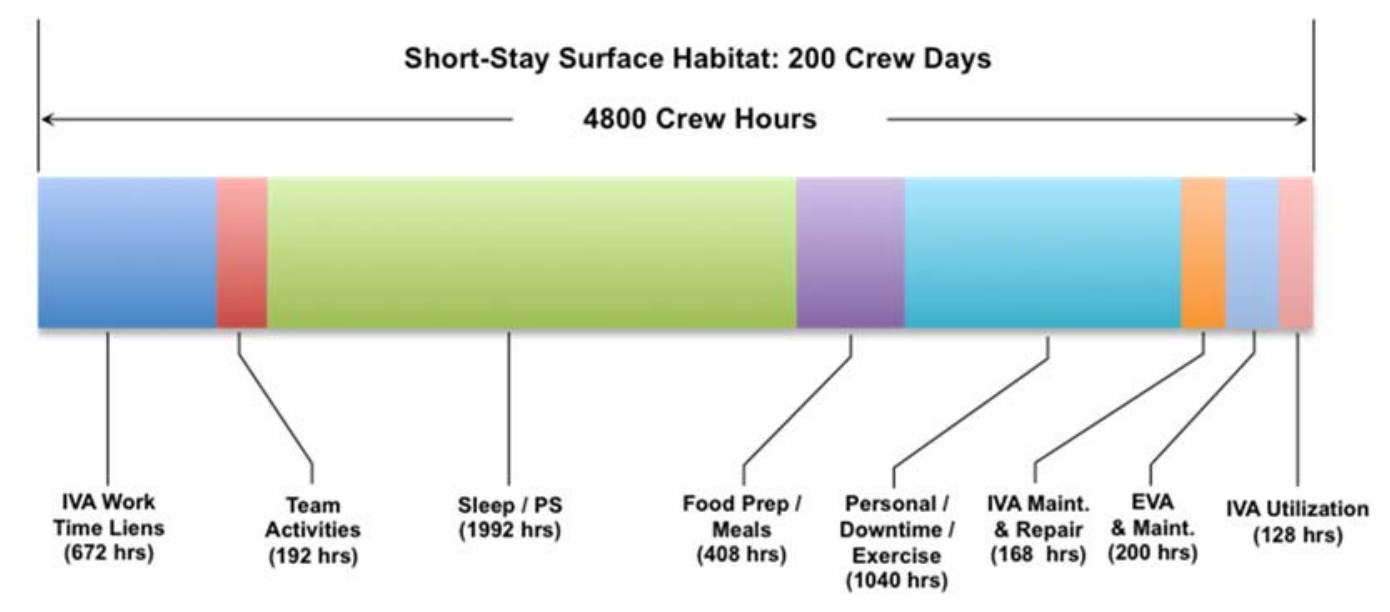

Figure 6: Short-Stay Surface Habitat Crew Time Allocations

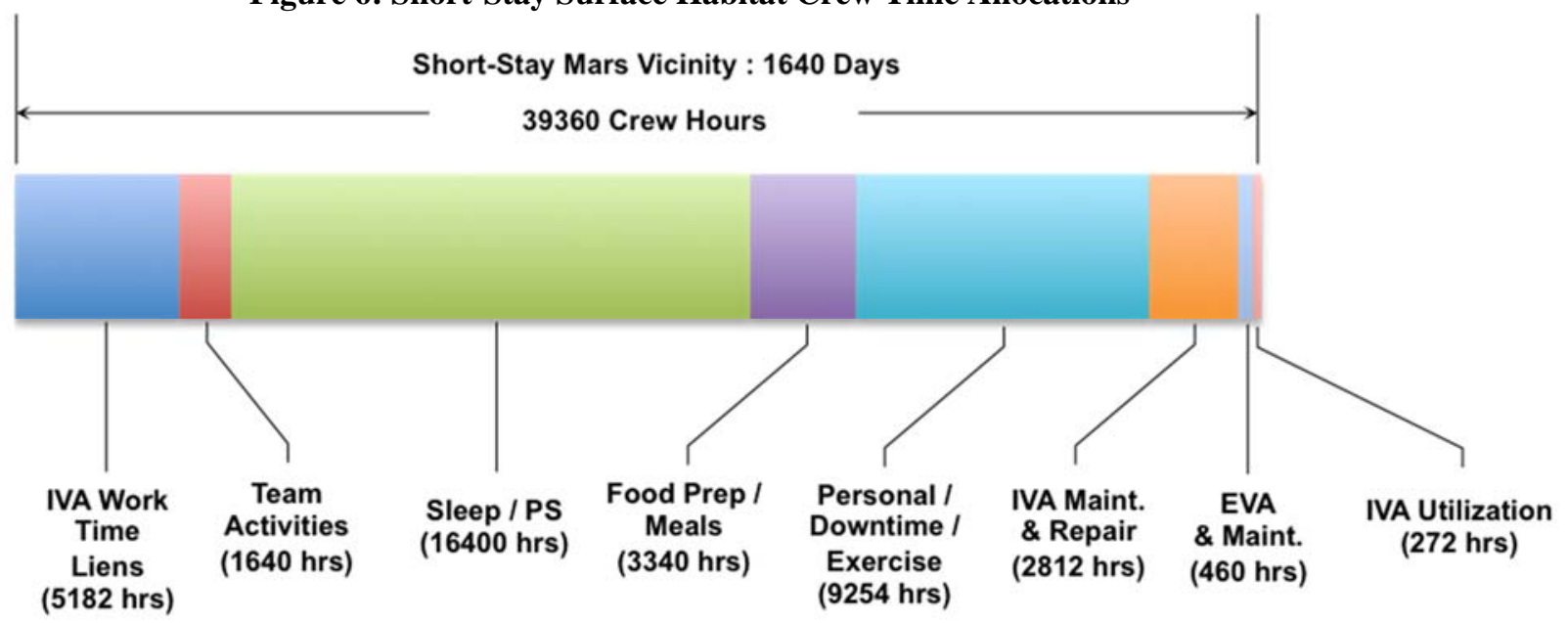

Figure 7: Short-Stay Mars Vicinity Crew Time Allocations

The long-duration surface architecture accommodates a maximum of 22, 15-day excursions (each using two SPRs with one crew member in each). This results in a total of 15,840 crew hours dedicated to excursion activities, 1,152 hours of which are allocated to SPR EVA. This equated to an average of approximately 1.75 hours per day per crew for EVA, while on excursion. It should be noted that the prioritization of excursion EVA imparts constraints on driving time while virtually eliminating SPR IVA utilization. During a single 15-day SPR excursion cycle, each SPR can be driven an average of 1.5 hours per day. The total of approximately 3.25 hours per day per crewmember combined for driving and EVA could be redistributed, with different variation between the two components. Time could also be redistributed to IVA utilization.

Long-stay outpost utilization activities were limited, given the extended periods in which the habiting crew was undergoing preparation for, recovering from, or executing an excursion activity. As a result, about $5 \%$ of outpost crew hours were available for utilization.

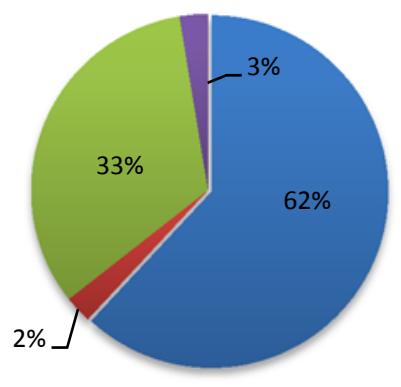

Inside Surface Hab

Hab EVA

Inside SPR

SPR EVA

Figure 8: Long-Stay Time Distribution 
Combining outpost and excursion phases, a total of 2,667 crew hours were allocated to utilization activities, including SPR drive time, during the 460-day surface stay. This represents $8.5 \%$ of total surface crew time that is available for utilization activities of any kind. Figure 8 provides the time distribution of the crew split between EVA, the central habitat, and rovers.

The 50 days of surface habitation for the short-duration stay can accommodate up to 320 crew hours of utilization at the surface. This results in $6.5 \%$ of total surface time being available for surface utilization. Similar to the long-stay case, the maximization of EVA opportunities significantly restricts the opportunities to complete IVA utilization tasks. As it is assumed there are no pressurized rovers in the short duration architecture, crew time is split only between surface EVA and the short duration habitat. For the short stay missions, no distinction is made between driving and EVA time.

The remaining 410 days of the short-stay case are spent within the transit habitat in Mars vicinity. The available utilization crew time is minimal; with time splits similar to the distribution observed during transit periods. A total of only 712 crew hours, about $2 \%$ of total crew hours, are available for utilization, including proposed tele-operation activities, while the short-stay crew remains in Mars vicinity. The time distribution for the short-stay architecture is provided in Figure 9.

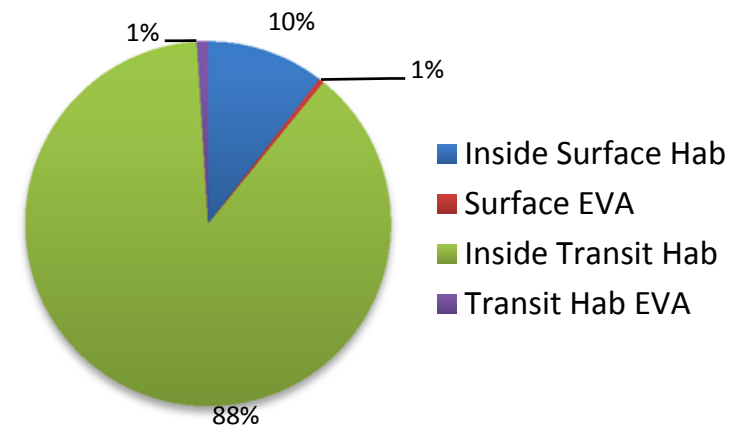

Figure 9: Short-Stay Time Distribution

For both surface architectures, a critical factor that limits utilization availability is reconditioning requirements, although the impact is varied between cases. While the short-stay mission requires a reconditioning period 23-days less than that of the long-stay (7 versus 30 days respectively) prior to commencement of EVA/excursion activities, the reconditioning duration constitutes a significantly larger portion of the total crewed surface duration. Nearly $20 \%$ of total crew time during the short duration stay is spent with the crew reconditioning compared to the $6.5 \%$ of the total crewed surface duration of the long stay mission. As a result neither crew is able to utilize the maximum allowable EVA at the surface.
Figure 10 shows time allocations for the in-transit duration of the missions between IVA and EVA activities.

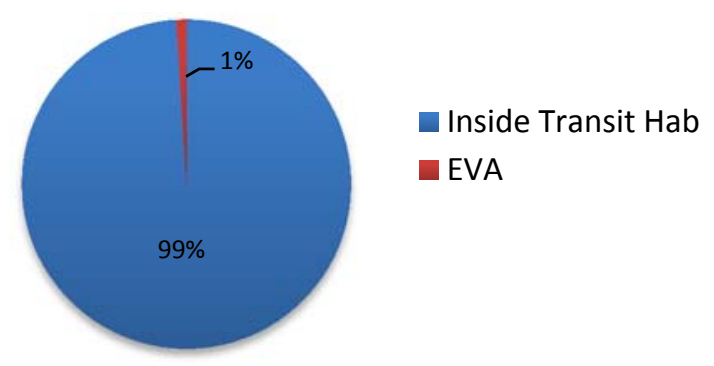

Figure 10: Transit Time Distribution

The time allocations for the in-transit segments are the same for both long and short duration missions. There is substantially less time available for utilization during the intransit phases. The largest contributor to the difference between transit and surface allocations are the crew exercise requirements. During transit periods, mitigation of microgravity exposure is significantly more challenging than in the hypogravity surface environment. Additionally, the much higher EVA frequency on the surface allow a nontrivial portion of crew physical demands to be satisfied in parallel with utilization activities. The result is a significant elevation in total crew hours allocated to exercise activities for the transit segments.

A detailed comparison of crew task work rate per mission phase is given in Table 4, along with historical ISS expedition data. Both surface cases will require a marked increase in crewmember work rate relative to ISS. The longstay architecture requires an additional 0.7 hours crew hours per day while the short-stay necessitates an additional 1.9 crew hours per day versus ISS experience. A nominal decrease of 0.1 hours per day was found for transit periods.

Table 4: Work Rate Per Mission Segment

\begin{tabular}{|l|c|c|}
\cline { 2 - 3 } \multicolumn{1}{c|}{} & $\begin{array}{c}\text { Mean Work } \\
\text { Hours/Dayl } \\
\text { Crew }\end{array}$ & $\begin{array}{c}\% \text { Change } \\
\text { from ISS } \\
\text { Baseline }\end{array}$ \\
\hline Historic ISS & 6.4 & \\
\hline Transit & 6.3 & $-1.25 \%$ \\
\hline $\begin{array}{l}\text { Long-Stay } \\
\text { Surface }\end{array}$ & 7.1 & $+12.5 \%$ \\
\hline $\begin{array}{l}\text { Short-Stay } \\
\text { Surface }\end{array}$ & 8.3 & $+29.5 \%$ \\
\hline
\end{tabular}




\section{Lien Improvement Opportunities}

The prominence of crew lien times as a constraint on utilization availability suggests that potential operational improvements could carry substantial benefits for exploration missions. However within the group of lien activities, only a subset of tasks are legitimate candidates for lien reduction. Decreased time allocations for items such as sleep, meals, personal, and R\&R time may present physiological and/or psychological ramifications that can jeopardize both crew health and mission success. For the purposes of this study, lien performance improvements will focus on work related tasks in which crew performance gains can be clearly applied and present minimal increases in crew risk.

Targeting only tasks in which improvements are viable, the sensitivity between lien reductions and utilization availability was evaluated. Figure 11 depicts the parametric relationship between changes in lien requirements (for tasks which can be modified) and available utilization time. Note that the data presented does not include architectural changes such as improved component reliability or EVA systems capability, only increases in the efficiency of the crew in completing tasks as prescribed by the baseline mission assumptions are included.

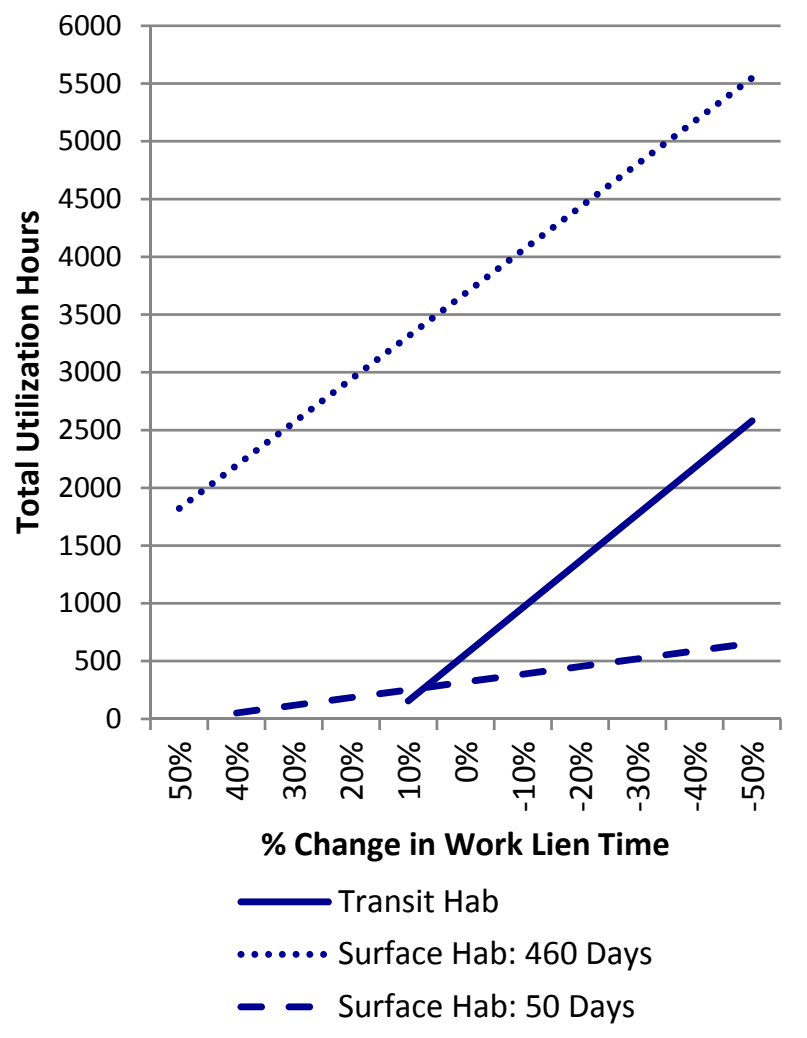

Steeper curves imply a more pronounced relationship between work lien time variations and utilization availability. The transit mission segment is the most sensitive to changes in crew work time. As utilization time is so limited under nominal conditions during transit segments, minor variations in lien time have the largest proportional impact on utilization crew hours. Additionally, the rate at which the transit utilization time trends to zero suggests that it is the least robust against crew work degradation. Crew work lien increases of only $10 \%$ would nearly eliminate all available utilization time. The long-stay surface case presents a similar sensitivity behavior to the transit segments, although the significantly larger pool of available utilization hours allows long-stay work lien operations to vary substantially and still allow for crew utilization time. The short-stay case presents the most stable response to variations in work lien time, although the low total utilization hours available to the crew on the surface carry less margin than the long-stay case. Work time increases of greater than $40 \%$ will eliminate surface utilization time for the short-stay architecture.

\section{Repair and Maintenance}

In addition to improvements to crew operational efficiency, changes to system architecture may offer the potential for decreased crew work requirements. The long durations of habitation systems operation required to support deep space exploration presents a multitude of challenges in terms of maintainability and repair. Highly complex habitation systems can reduce consumables mass but will likely place additional life-critical repair and maintenance loads on the crew. Even under the mission architecture presented in this study - evolutionary systems based on ISS heritage - the repair and maintenance time required of the crew is substantial. Using the probabilistic Monte Carlo simulation procedure described in Section 2, the expected repair times to maintain a transit and surface habitat were developed. The results of the maintenance and repair time analysis are presented in Table 5.

Table 5: Crew Repair Time Results

\begin{tabular}{|l|c|c|}
\cline { 2 - 3 } \multicolumn{1}{c|}{} & Transit Habitat & Surface Habitat \\
\hline $\begin{array}{l}\text { Mean Repair } \\
\text { Hours/Week Per } \\
\text { Crew }\end{array}$ & 5.0 & 7.5 \\
\hline $\begin{array}{l}\text { Mean Maintenance } \\
\text { Hours/Week Per } \\
\text { Crew }\end{array}$ & 7.0 & 8.5 \\
\hline $\begin{array}{l}\text { Mean R\&M Hours/ } \\
\text { Week Per Crew }\end{array}$ & 12.0 & 16.0 \\
\hline
\end{tabular}

Figure 11: Work Lien Variation 


\section{Repair and Maintenance Sensitivity}

A similar sensitivity analysis was conducted to assess perturbations in repair performance. Figure 12 depicts the results of this sensitivity study, with the total available utilization crew hours versus component reliability performance, measured as Mean Time Between Failure (MTBF), provided.

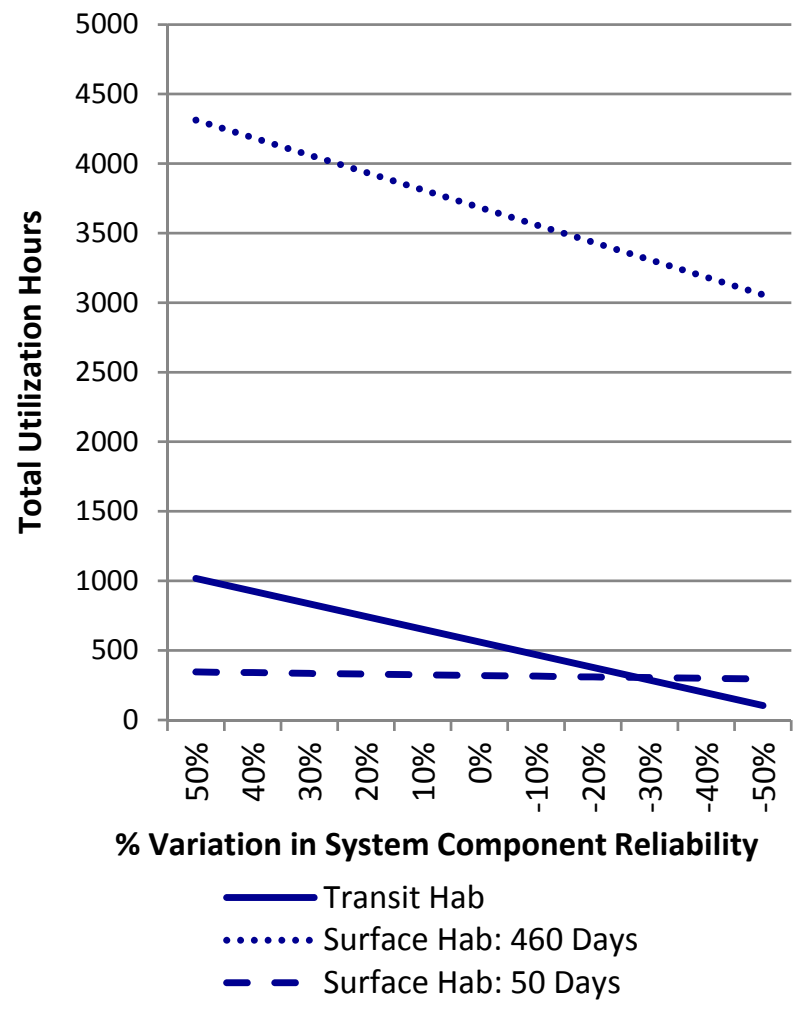

\section{Figure 12: System Component Reliability Variation}

Utilization sensitivity to system reliability between cases can be directly ascertained from the slopes of the 3 curves. The transit and long-stay architectures present similar responses to variations in system component reliability. Improvements in system reliability yield significant increases in the amount of time available for utilization. Utilization variations are substantially less pronounced for the short-stay surface case. This reduced response sensitivity can be attributed to the lower system complexity of the short-stay habitat architecture, which limits the total time allocated to repair and maintenance.

While the transit and long-stay surface habitats share similar rates of response to variations in reliability, the transit architecture presents a critical behavior not displayed in the long-stay habitat. Due to the low utilization margin nominally associated with the transit architecture, it is markedly less robust against decreases in system performance. For decreases in component reliability greater than $50 \%$, the time margin available for utilization is effectively non-existent. This raises concerns over the safety of the in-transit mission segments. If reliability is significantly worse than predicted, there may not be sufficient time for the crew to complete repair operations without reducing other crew time liens.

\section{Surface Operations Sensitivity}

Both surface architectures were initially assessed under the assumption of a maximum of 24 EVA hours/week per crewmember with one day of rest within that same 7-day period. Current HAT Mars surface destination team assumptions suggest that this constraint may be relaxed for short-stay mission architectures. The impacts of varying EVA constraints are shown in Figure 13.

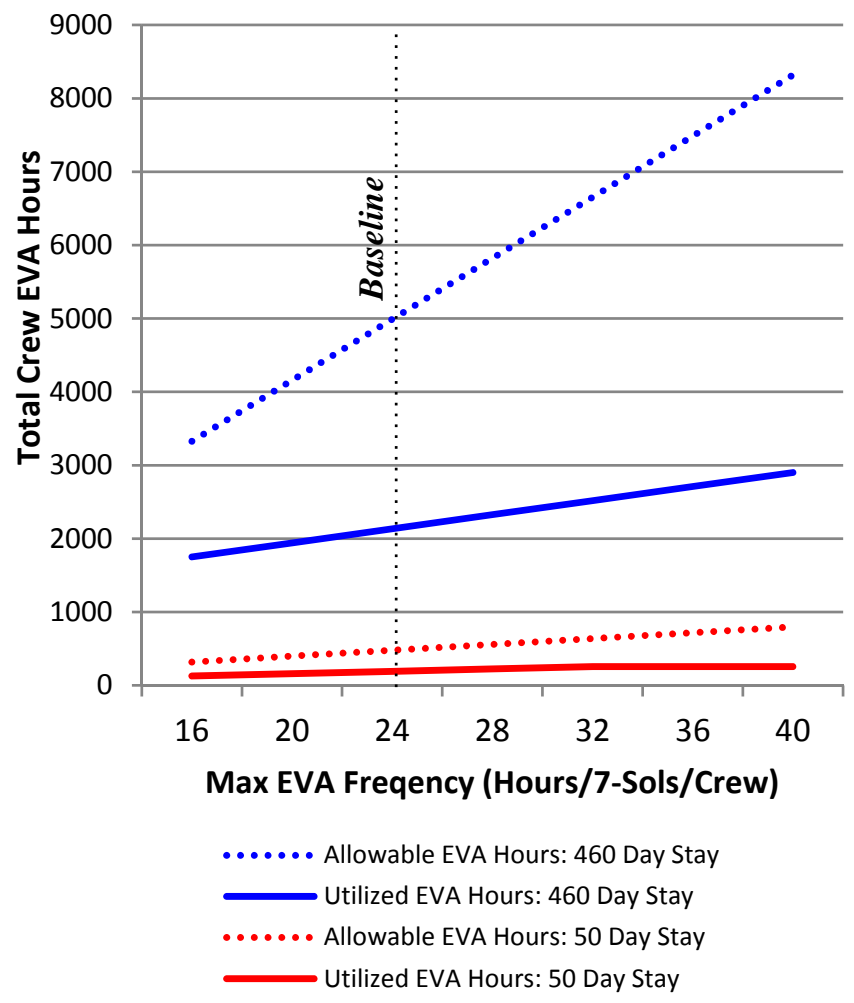

Figure 13: EVA Frequency Variation

Modifying the EVA constraints does little to enable additional utilization opportunities for the short-stay case and produces marginal gains for the long-stay case. The nearly horizontal Utilized EVA curve for the short-stay case emphasizes lack of impact that EVA constraints have on the utilized EVA for the short-stay missions. This is because the total EVA time on surface missions is generally constrained by time availability rather than EVA limits.

For the long-stay case, changes in EVA limits can impact the total amount of EVA that can be conducted. However, continuous EVA rates exceeding the nominal 24 hours per 7-day period are aggressive, likely exceeding reasonable expectation for crew performance. 
The impacts of changing the distribution between driving time and EVA time during excursions was also evaluated. For the long-stay case, including drive time, a total of 2171 crew hours are available for utilization during SPR excursions. While the baseline solution sought to prioritize EVA time (1152 hours) over SPR drive time (1019 hours), these allocations may be directly traded if so desired. The relationship between total EVA and SPR drive time is provided in Figure 14.

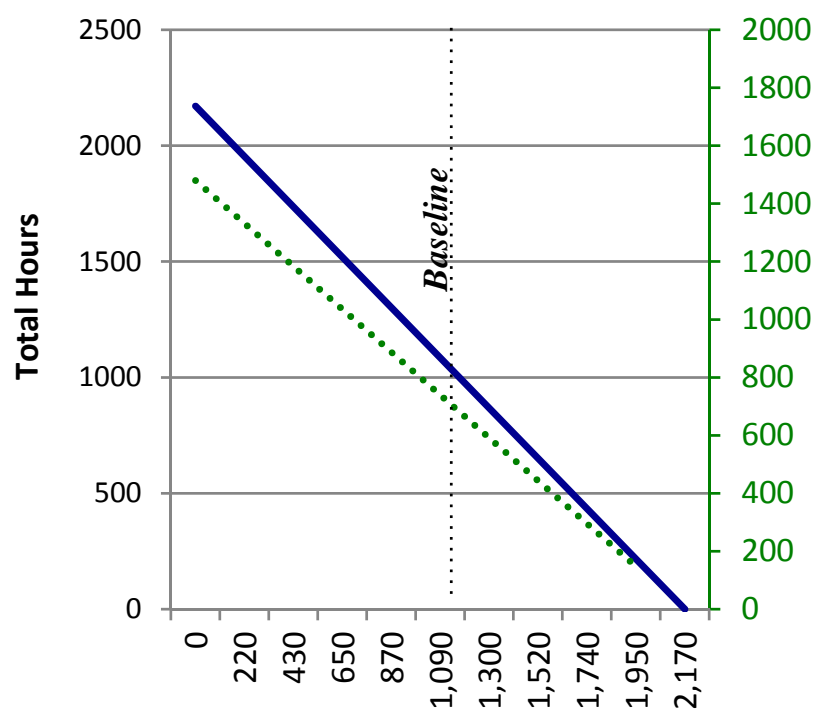

Total SPR EVA Crew Hours

Total SPR Drive Hours

...... Mean Traversal Distance Per Excursion

Figure 14: SPR EVA Hours Versus SPR Drive Time

Assuming a mean SPR traversal velocity of $15 \mathrm{~km}$ per hour, every 3-hour reduction to total SPR EVA crew time results in an additional $1 \mathrm{~km}$ of traversal distance is added to each 15-day excursion. Thus a reduction of 750 SPR EVA hours would allow for an additional 250 kilometers of surface traversal during each 15-day excursion.

As previously discussed, the maximization of SPR excursion and EVA time eliminates a large portion of IVA utilization opportunities at the outpost. Excursion time may be reduced to increase utilization time at the outpost. The results of this trade are presented in Figure 15.

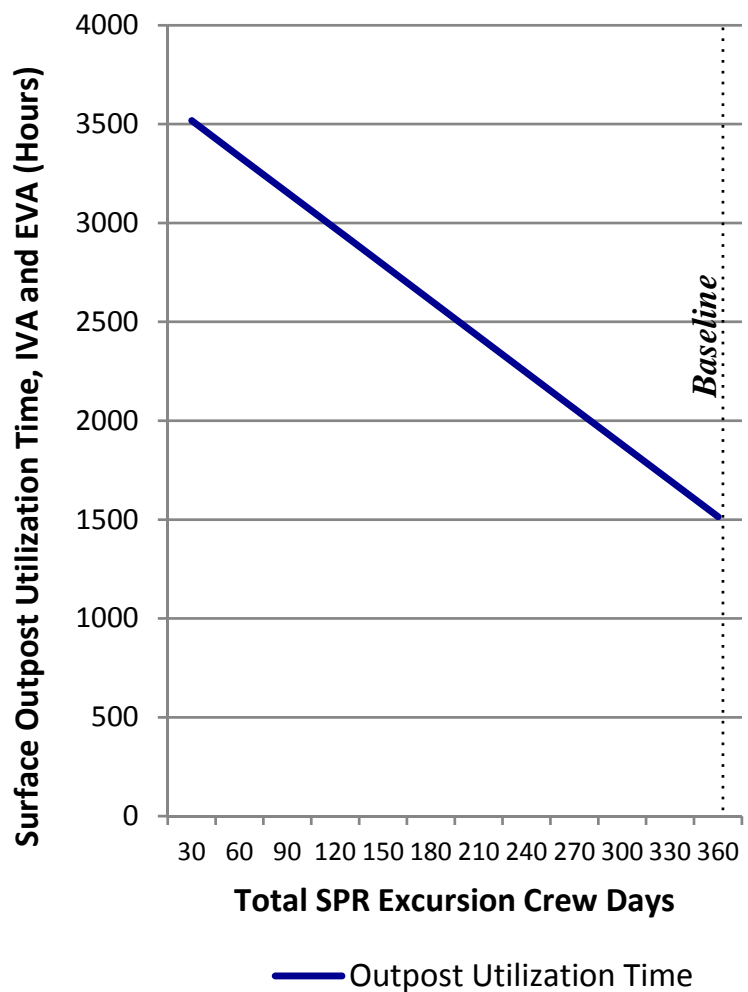

Figure 15: SPR Excursion Duration Versus Outpost
Utilization

In the initial assessment, 22 X 15 day of excursion time, with two crew, allows for 1515 hours of outpost utilization time, including both IVA and EVA. To enable a four hour per week outpost IVA utilization rate per crewmember, a significant $25 \%$ reduction in excursion days is required.

\section{CONCLUSIONS}

An initial integrated assessment of crew time requirements for Mars destination exploration missions suggests utilization opportunities may be limited by required crew overhead activities. A comprehensive assessment of crew operations indicates that the influence of crew time liens, habitation systems maintenance, and EVA constraints, along with the limited crew size, demonstrate that reductions in the time required for the crew to perform certain activities will have to be achieved in order to increase the amount of utilization on a Mars mission. In addition, the results indicate that crewed exploration architectures may pose new concerns regarding crew operational loading, which will require novel approaches to mitigate the elevated task loads imparted on an exploration crew.

Crew liens are the primary driver of time allocations, constituting over $90 \%$ and $80 \%$ of total available crew time for transit and surface operations, respectively. As a 
significant proportion of total liens consist of non-work related activities necessary to sustaining crew physical and psychological health, there exist only a small subset of liens reasonably viable for performance improvements.

Transit durations to and from Mars, which likely exceed 300 days under the current propulsive architecture assumptions, present new physiological risks that require additional countermeasures to ensure crew health. The additional of these countermeasures will require substantially larger crew time investments in exercise and medical activities, limiting available utilization time during transit.

The assessment confirmed the importance of repair and maintenance time requirements to overall exploration crew operations. The time required to maintain and repair exploration habitation systems plays a prominent role in limiting available time for utilization. Additional crew time availability for utilization could be made available through improvements in component reliability and/or repair efficiency. As expected, reducing the expected time to repair for system components provides direct gains to utilization time.

In addition, the maintenance and repair time estimates used in the assessment were taken at the $90 \%$ confidence level. This means that there is a probability that the actual repair and maintenance times experienced on the mission could be greater than allocated for. Because the actual time requirements will be based on random failures, there is no way to definitively predict repair times prior to the mission. If the actual required maintenance and repair time were greater than expected, there would be major impacts on the mission. During surface phases, the already limited amount of utilization time would be further reduced. During transit phases there is also the possibility that there would not be enough time available to complete all maintenance and repair activities without reducing time allocated to other crew liens.

Simpler, low complexity systems that are easier to repair may allow for more efficient use of crew time when compared to the high complexity associated with high closure exploration systems architectures. Lower closure systems do present tradeoffs with respect to logistics requirements however, and thus further investigations into the trade space may be necessary.

For surface operations, an evaluation of the long stay mission structure revealed significant restrictions to both IVA and EVA utilization availability. Since surface operations occur in a hypogravity environment and there will be an expected increase in surface EVA rates, crew exercise requirements will be less while on the surface. Therefore, the time available for utilization is somewhat greater than during the in-transit segments.

However, EVA performance becomes a critical factor in determination of available utilization on the surface. The usage dependent nature of EVA maintenance resulted in rapid growth in EVA maintenance allocations as EVA rates were increased. At the assumed maximum EVA rate of 24 hours/week per crewmember, EVA maintenance requirements were non-trivial, reducing SPR utilization by an average of 2 hours per week. More conservative maintenance estimates resulted in even greater impacts.

Given the already restricted total pool of utilization hours available to the crew at the surface, maximization of SPR EVA presents a major tradeoff, particularly with respect to SPR drive and outpost utilization capabilities. As a result, an almost one-to-one sensitivity between surface EVA, SPR drive, and outpost utilization was demonstrated, with EVA time directly reducing crew time available for SPR driving and outpost utilization. Greater excursion distances will require either significant reductions in SPR EVA or a relaxation of crew EVA recovery requirements.

All the cases analyzed assume a crew size of four, with all four crew descending to the surface. Increases in crew size, while imposing major changes to the exploration architecture, would potentially make substantially more time available for utilization activities.

\section{FUTURE WORK}

The baseline mission structure presented in this paper represents one possible set of architectural assumptions each for transit, short, and long duration mission phases. There are numerous potential changes to these assumptions that could be assessed in the future to evaluate exploration crew time. Potential changes include:

- Increases in crew size and Apollo-style split crew structures.

- Use of SPR architecture for short duration surface stays rather than a singular outpost.

- Crew performance degradation over the course of the mission duration.

- Variations in excursion objectives and SPR traversal performance.

- Changes in habitat atmospheres to allow for optimized EVA egress/ingress.

- Use of Low-Latency Telerobotics and robotic systems to reduce crew operational requirements.

Furthermore, a detailed investigation into the mass tradeoffs associated with various levels of habitation systems closure could be developed to provide context to any improvements in maintenance and repair time allocations.

Additional outpost tasking must also be investigated to evaluate impacts to utilization capabilities. Such tasks include:

- $\quad$ Site preparation

- ISRU support

- $\quad$ Surface propellant transfer 
- MAV preparation/maintenance

- Regolith moving for radiation protection

Future assessments will require working with numerous subject matter experts, including EVA, ECLSS, technologists, and surface architecture teams to identify where operational improvements may be made.

In addition, it will also be necessary to perform a dynamic assessment of crew time availability for exploration missions. The current ICOM assessment allocates total crew hours over individual mission segments and surface excursions but does not look at crew time loading on a dayto-day basis. While many crew activities are predictable and will be spread relatively evenly over mission segments, certain activities, such as system repair are unpredictable and more stochastic in nature. There is the potential that the need for repair activities at certain times could overwhelm the crew.

\section{ACKNOWLEDGEMENTS}

The authors acknowledge Natalie Mary (NASA Johnson Space Center) for sharing her insight and knowledge into current Mars EVA architectures and assumptions. The authors also acknowledge Larry Toups (NASA Johnson Space Center) and Stephen Hoffman (SAIC/Johnson Space Center) for sharing their insight and current work regarding crew destination operations architectures and assumptions. The authors acknowledge Patrick Troutman (NASA Langley Research Center) for sharing his knowledge into current Mars exploration campaign architectures and assumptions.

\section{REFERENCES}

[1] NASA, "International Space Station Timelines," http://www.nasa.gov/mission_pages/station/timelines/\#.V DU3iIfXN74, 2000-2014.

[2] Stromgren, C., Terry, M., Cirillo, W., Goodliff, K. and Maxwell, A., "Design and Application of the Exploration Maintainability Analysis Tool,” AIAA Space 2012 Conference and Exposition, AIAA-2012-5323, Pasadena, CA, September 2012.

[3] Stromgren, C., Terry, M., Mattfeld, B., Cirillo, W., Goodliff, K., Shyface, H., and Maxwell, A., "Assessment of Maintainability for Future Human Asteroid and Mars Missions," AIAA Space 2013 Conference and Exposition, AIAA-2013-5328, Pasadena, CA, September 2013.

[4] Discussion with NASA Destination Operations Team regarding Mars surface destination operations, 2014.

[5] Discussion with Mary, N, and NASA Destination Operations Team regarding current EVA assumptions, 2014.

[6] Stromgren, C., NASA Strategic Analysis Team, "Lunar Surface Systems Crew Time Analysis Overview," Presentation, January 21, 2009. 


\section{BIOGRAPHY}

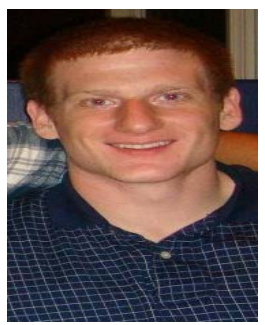

Bryan Mattfeld received bachelor's degrees both in Aerospace Engineering and Mathematics from Virginia Polytechnic and State University in Blacksburg, Virginia in 2013. He has 4 years experience in space systems engineering and risk analysis. His work includes the development and analysis of space systems and mission design models. He currently works for Binera, Inc. in Silver Spring, Maryland and is an AIAA member.

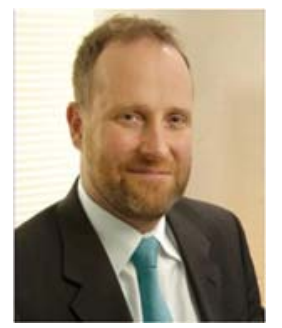

Chel Stromgren currently serves as the Chief Scientist of Binera, Inc. Risk Analytics Division. In this role, Mr. Stromgren leads the development of probability and risk-based strategic models and strategic analysis of complex system development. Mr. Stromgren has supported NASA in the analysis of Space Shuttle and International Space Station operations in the post-Columbia environment and has led the development of strategic campaign models for the lunar exploration initiatives. He holds a Bachelor of Science degree in Marine Engineering and Naval Architecture from the Webb Institute and a Master of Science degree in Systems Management from the Massachusetts Institute of Technology.

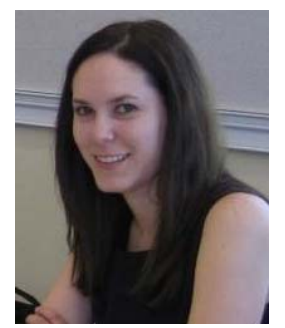

Hilary Shyface serves as a systems engineer for Analytical Mechanics Associates, Inc, in Hampton, VA. She performs campaign analysis for human exploration and conceptual element sizing in support of NASA's Space Mission Analysis Branch. She has a Bachelor of Science in Mechanical Engineering from the University of Nevada, Las Vegas and a Master of Engineering in Systems Engineering from the University of Virginia

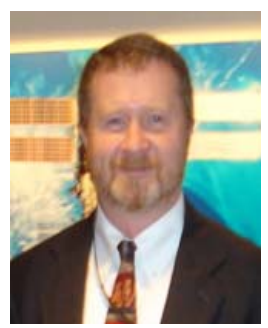

William Cirillo currently serves as a Senior Researcher at NASA Langley Research Center in Hampton, Virginia, where he has worked for the past 20 years in the area of Human Space Flight Systems Analysis. This has included studies of Space Shuttle, International Space Station, and Human Exploration beyond low Earth orbit. In 2005, Mr. Cirillo served at NASA Headquarters as a core member of the Exploration Systems Architecture Study (ESAS) team, where he was responsible for studying the use of Ares I/Orion in meeting future ISS crew and logistics transportation needs. Mr. Cirillo currently leads a team of analysts in assessing at a strategic and tactical level the manifesting of assembly and logistics flights human exploration beyond low Earth orbit.

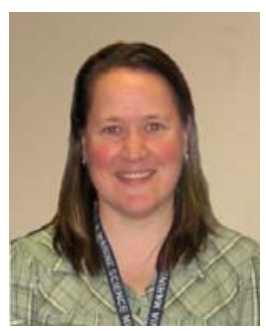

Kandyce Goodliff is an aerospace engineer at NASA Langley Research Center in Hampton, VA, with the Space Mission Analysis Branch (SMAB). Her primary roles as a systems analyst for SMAB are conceptual design and sizing of human and robotic spacecraft, mission and spacecraft analysis, and campaign analysis for human exploration. She has a Bachelor of Science in Aerospace Engineering from Embry-Riddle Aeronautical University and a Master of Science in Mechanical Engineering from the George Washington University. 
